\title{
Urinary biomarkers are associated with incident cardiovascular disease, all-cause mortality and deterioration of kidney function in type 2 diabetic patients with microalbuminuria
}

\author{
Bernt Johan von Scholten ${ }^{1}$ - Henrik Reinhard ${ }^{1}$ - Tine W. Hansen ${ }^{1}$ • Jens Oellgaard ${ }^{1,2,3}$. \\ Hans-Henrik Parving ${ }^{4}$ • Peter K. Jacobsen ${ }^{5}$ • Peter Rossing ${ }^{1,6,7}$
}

Received: 2 November 2015 / Accepted: 22 February 2016 /Published online: 31 March 2016

(C) Springer-Verlag Berlin Heidelberg 2016

\begin{abstract}
Aims/hypothesis We evaluated two urinary biomarkers reflecting different aspects of renal pathophysiology as potential determinants of incident cardiovascular disease (CVD), all-cause mortality and a reduced estimated GFR (eGFR) in patients with type 2 diabetes and microalbuminuria but without clinical features of coronary artery disease.

Methods In a prospective study of 200 patients, all received multifactorial treatment. Baseline measurements of urinary hepatocyte growth factor (HGF) and adiponectin were available for 191 patients. Cox models were adjusted for sex, age, LDL-cholesterol, smoking, $\mathrm{HbA}_{1 \mathrm{c}}$, plasma creatinine, systolic $\mathrm{BP}$ and urinary AER (UAER). The pre-defined endpoint of chronic kidney disease progression was a decline in the eGFR
\end{abstract}

Electronic supplementary material The online version of this article (doi:10.1007/s00125-016-3937-0) contains peer-reviewed but unedited supplementary material, which is available to authorised users.

Bernt Johan von Scholten bjos@steno.dk

1 Steno Diabetes Center, Niels Steensens Vej 1, 2820 Gentofte, Denmark

2 Faculty of Health Sciences, University of Southern Denmark, Odense, Denmark

3 Department of Endocrinology, Slagelse Hospital, Slagelse, Denmark

4 Department of Endocrinology, Rigshospitalet, University of Copenhagen, Copenhagen, Denmark

5 Department of Cardiology, The Heart Center, Rigshospitalet, Copenhagen, Denmark

6 The Novo Nordisk Foundation Center for Basic Metabolic Research, University of Copenhagen, Copenhagen, Denmark

7 Faculty of Health, Aarhus University, Aarhus, Denmark of $>30 \%$ during follow-up. HRs per 1 SD increment of logtransformed values are presented.

Results Patients had a mean \pm SD age of $59 \pm 9$ years with a median (interquartile range) UAER of 103 (39-230) mg/24 h. During a median 6.1 years of follow-up, there were 40 incident CVD events, 26 deaths and 42 patients reached the pre-defined chronic kidney disease progression endpoint after 4.9 years (median). Higher urinary HGF was a determinant of CVD in unadjusted (HR 1.9 [95\% CI 1.3, 2.8], $p=0.001$ ) and adjusted (HR 2.0 [95\% CI 1.2, 3.2], $p=0.004$ ) models, and of all-cause mortality in unadjusted (HR 2.3 [95\% CI 1.3, 3.9], $p=0.003$ ) and adjusted (HR 2.5 [95\% CI 1.3, 4.8], $p=0.005$ ) models. A higher adiponectin level was associated with CVD in unadjusted (HR 1.4 [95\% CI 1.0, 1.9], $p=0.04$ ) and adjusted (HR 1.4 [95\% CI $1.1,2.3], p=0.013$ ) models, and with a decline in the eGFR of $>30 \%$ in unadjusted (HR 1.6 [95\% CI 1.2, 2.2], $p=0.008$ ) and adjusted (HR 1.5 [95\% CI 1.1, 2.2], $p=0.007$ ) models.

Conclusions/interpretation In patients with type 2 diabetes and microalbuminuria receiving multifactorial treatment, higher urinary HGF was associated with incident CVD and all-cause mortality, and higher adiponectin was associated with CVD and deterioration in renal function.

Keywords Adiponectin - Cardiovascular disease · Hepatocyte growth factor - Macrovascular disease . Microalbuminuria $\cdot$ Type 2 diabetes $\cdot$ Urinary biomarkers

$\begin{array}{ll}\text { Abbreviations } \\ \text { CAC } & \text { Coronary artery calcium } \\ \text { CAD } & \text { Coronary artery disease } \\ \text { CKD } & \text { Chronic kidney disease } \\ \text { CVD } & \text { Cardiovascular disease } \\ \text { eGFR } & \text { Estimated GFR } \\ \text { HGF } & \text { Hepatocyte growth factor }\end{array}$


IDI Integrated discrimination improvement

NT-proBNP N-terminal pro-brain natriuretic peptide

RAAS Renin-angiotensin-aldosterone system

rIDI Relative IDI

ROC Receiver operating characteristic

UAER Urinary AER

\section{Introduction}

Albuminuria is considered one of the best available risk factors of renal disease $[1,2]$ and cardiovascular disease (CVD) [3, 4]; reductions in albuminuria have been linked to both renal and cardiovascular protection $[1,5]$. The wide implementation of renin-angiotensin-aldosterone system (RAAS) blocking treatment in type 2 diabetes has made a major contribution to slowing disease progression; however, patients with type 2 diabetes are still at a high risk of both renal and cardiovascular complications. There is therefore an ongoing search for new biomarkers to provide additional prognostic information beyond that provided by albuminuria and to potentially provide new therapeutic targets.

In this study, we pre-selected two urinary biomarkers representing different aspects of renal pathophysiology: (1) hepatocyte growth factor (HGF), a marker linked to renal tubular epithelial cell regeneration [6]; and (2) adiponectin, a marker of early glomerular vascular damage [7]. The role of HGF in the cardiovascular system has been investigated previously. Studies have reported that HGF is markedly elevated in patients with end-stage renal disease, and may be considered a novel vascular modulator in humans [8]. In a cross-sectional study, we previously found urinary adiponectin excretion to be positively related to albuminuria in type 1 diabetes [9]. Moreover, prospective studies have shown that high urinary adiponectin is associated with the development of end-stage renal disease [10, 11]. The association between these urinary markers, incident CVD and all-cause mortality, when adjusted for levels of $\mathrm{N}$ terminal pro-brain natriuretic peptide (NT-proBNP) and the coronary artery calcium (CAC) score is, however, unknown.

The aim of this prospective study was to evaluate two preselected urinary biomarkers as determinants of incident CVD and all-cause mortality in patients who had type 2 diabetes and microalbuminuria but no clinical features of coronary artery disease (CAD), and were receiving multifactorial treatment. Deterioration in the estimated GFR (eGFR), as a measure of chronic kidney disease (CKD) progression, was also assessed.

\section{Methods}

\section{Participants and study procedure}

At the Steno Diabetes Center, we identified a cohort of 200 outpatients with type 2 diabetes treated in a secondary care setting from January 2007 to February 2008 [12]. All patients received treatment with multifactorial intervention, consisting of glycaemic, lipid and BP control, as well as antithrombotic therapy and lifestyle modification in line with the findings of the Steno-2 Study [13]. Patients were included if they: (1) were outpatients with type 2 diabetes defined according to WHO criteria; (2) had no history of CAD or other cardiac disease and no symptoms suggestive of cardiac disease, as assessed from their medical records and patient interviews and questionnaires; and (3) had a persistent (two out of three consecutive measurements) urinary AER (UAER) of $>30 \mathrm{mg} / 24 \mathrm{~h}$.

A written invitation was sent to 613 consecutive patients ( $69 \%$ men; mean \pm SD age, $47 \pm 8$ years). A total of 72 patients declined participation. After telephone interview or examination in the outpatient clinic, 341 patients were excluded because one or more of the following characteristics were present: (1) normal or non-persistent elevated UAER $(n=52)$; (2) symptoms/signs or a history of heart disease, including Q waves in 12-lead ECGs $(n=180)$; (3) relative contraindications to computed tomography angiography or coronary angiography, including abnormal plasma creatinine levels $(n=86)$; (4) physical or mental disability $(n=10)$; or (5) malignancy $(n=13)$. Thus, the final study population comprised $200 \mathrm{pa}-$ tients. A detailed flow chart of the selection of the study population is shown in Fig. 1.

In the absence of data from other investigations using the biomarkers, this was an exploratory study using the sample size available. The study complied with the Declaration of Helsinki, the research protocol was approved by the local ethics committee and all patients gave written informed consent.

\section{Biochemical and other analyses}

The urinary biomarkers HGF and adiponectin were measured by ELISA (Roche Diagnostics, Basel, Switzerland) in a single $24 \mathrm{~h}$ urine sample from each patient. Immediately after collection, the urine was clarified by centrifugation, frozen at $-80^{\circ} \mathrm{C}$ and stored in a research biobank for analysis immediately after the last study patient was examined. The maximal storage time of the samples prior to analysis of both biomarkers was 13 months. The values were corrected for the urinary creatinine concentration. The biomarkers were not measured in duplicate. For HGF, the intra-assay and interassay CVs were $<10 \%$ and $<12 \%$, respectively. The high molecular weight isoform of adiponectin, which is the most biologically active, was measured: the intra-assay and interassay $\mathrm{CVs}$ were $<10 \%$ and $<15 \%$, respectively.

UAER was measured in $24 \mathrm{~h}$ collected urine samples using an enzyme immunoassay (Vitros, Raritan, NJ, USA). Current smoking was defined as one or more cigarettes, cigars or pipes per day. Plasma NT-proBNP was analysed by immunoassay and CAC scanning was performed as previously described [12]. The total Agatston CAC score [14], including intimal 
Fig. 1 Flow chart showing the selection of the final study population. CT, computed tomography

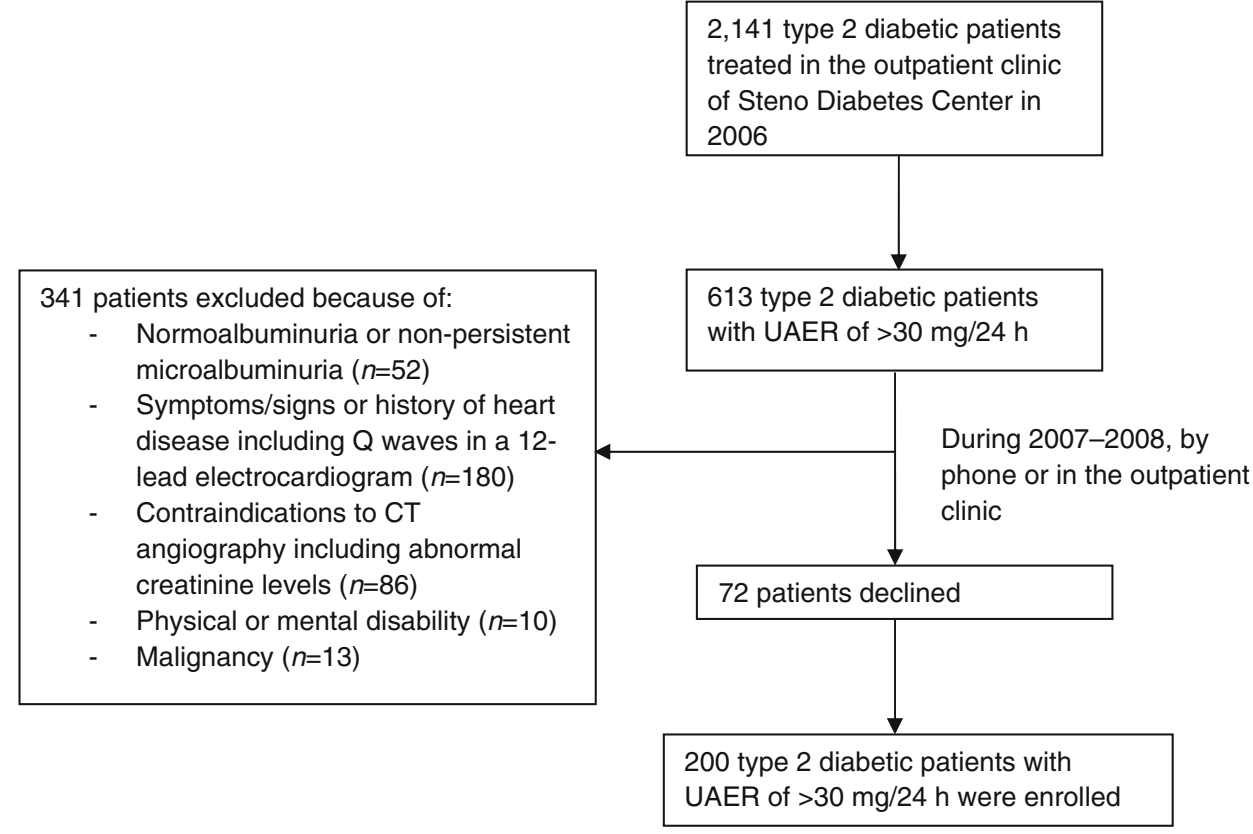

and medial calcification of the left main, left anterior descending artery, circumflex artery and right coronary artery, was determined for each patient.

\section{Follow-up}

On 1 January 2014, we traced all patients through the Danish National Death Register and the Danish National Health Register. No patients were lost to follow-up. For deceased patients, we obtained information on the date and cause of death. All deaths were classified as CVD unless an unequivocal non-CVD cause was established. Information on hospital admission, including for non-fatal CVD, was obtained from the Danish National Health Register.

We pre-defined a broad primary endpoint consisting of cardiovascular mortality, non-fatal myocardial infarction (ICD-10 codes I20-I25), stroke (ICD-10 codes I61 or I63), ischaemic cardiovascular disease (ICD-10 code I70) and heart failure (ICD-10 code I50). None of the patients were classified according to the results of baseline investigations (i.e. elevated CAC or NT-proBNP). For patients who experienced multiple endpoints, the analysis included only the first endpoint. The secondary endpoint was all-cause mortality.

Of the 200 patients originally included in the study, 177 $(88.5 \%)$ were followed for a median (interquartile range [IQR]) of 4.9 (3.8-5.4) years after baseline examination, with a yearly measurement of plasma creatinine levels for calculating the eGFR by applying the Chronic Kidney Disease Epidemiology Collaboration (CKD-EPI) equation [15]. Changes in the eGFR were evaluated from baseline until the last available measurement. The last patient had the latest measurement performed in March 2013. A decline in the eGFR of $>30 \%$ at any time point was the pre-defined endpoint of CKD progression, as proposed by Coresh et al [16]. Baseline values of urinary HGF and adiponectin were available for 191 patients (96\%).

\section{Statistical analyses}

The distribution of the urinary biomarkers is shown in electronic supplementary material (ESM) Fig. 1. Based on their graphical distribution and a significant result in the ShapiroWilk test ( $p \leq 0.001$; indicating non-normal distribution), both biomarkers were log-transformed using $\log _{10}$.

All non-normally distributed variables are summarised as the median with IQR. All other continuous variables are summarised as means $\pm \mathrm{SD}$, and categorical variables are reported as total numbers with corresponding percentages. Differences in potential confounders between quartiles of the two urinary biomarkers were tested with the trend test for continuous variable, and the $\chi^{2}$ test for categorical variables.

Cox proportional hazards analyses were applied to compute the HRs with 95\% CIs for the CVD endpoint and allcause mortality, per SD increment of log-transformed values of the urinary biomarkers.

First, we used unadjusted models (Model 1) to determine whether there was any association between the analysed biomarkers and our pre-defined endpoints. Subsequent adjustment in all analyses included traditional CVD/renal risk factors based on existing evidence: sex, age, LDL-cholesterol, smoking, $\mathrm{HbA}_{1 \mathrm{c}}$, plasma creatinine, systolic BP and UAER (Model 2). For the CVD endpoint and all-cause mortality, we used models including the previously listed factors plus the NT-proBNP level and CAC score (Model 3). This model was included 
because the NT-proBNP level and CAC score were previously shown to be powerful risk markers in this cohort [17].

To minimise the risk of instability in the models, we also used a model including only those covariates related to each of the endpoints. These covariates were selected based on backward elimination, using $p<0.05$ as the threshold for retention. For the CVD endpoint, age and LDL-cholesterol level were included as covariates; for all-cause mortality, smoking was the only covariate included; and for the CKD progression endpoint, the UAER and plasma creatinine level were included.

Finally, we applied the Kaplan-Meier failure function to compare the risks of the CVD endpoint and all-cause mortality according to the median HGF level.

To examine the potential additional predictive ability of each urinary biomarker over traditional CVD risk factors, we plotted receiver operating characteristic (ROC) curves based on logistic regression models and calculated the AUC in a model including only the traditional CVD/renal risk factors and in the same model with each of the biomarkers added.

Next, we calculated the integrated discrimination improvement (IDI) statistics [18] to compare the overall improvement in sensitivity and specificity between two models: the model including traditional CVD/renal risk factors alone vs the model including the urinary biomarker. In other words, the IDI assesses the ability of the new biomarkers to improve the average sensitivity without sacrificing average specificity. The IDI may be a more powerful way to demonstrate improved diagnostic performance than the AUC [18]. The results of the IDI can be difficult to interpret, so we have also provided a relative IDI (rIDI), defined as the increase in discrimination slope of the model that includes the traditional CVD/renal risk factors plus the urinary biomarker divided by the slope of the model that includes only the traditional CVD/renal risk factors. The biomarker contribution can be more easily interpreted using the rIDI (reported as a percentage) than the IDI [18].

A two-tailed $p$ value of $<0.05$ was considered significant. Statistical analyses were performed using SPSS for Windows (version 20.0, Chicago, IL, USA), SAS software (version 9.3, SAS Institute, Cary, NC, USA) and Stata/IC version 14.0 for Windows (StataCorp, College Station, TX, USA).

\section{Results}

\section{Patient characteristics}

Table 1 lists the baseline characteristics of patients, categorised according to the occurrence (or not) of the three pre-defined endpoints. The total population $(n=200)$ included $151(76 \%)$ men, with a mean \pm SD age of $59 \pm 9$ years, known diabetes duration of $13 \pm 7$ years and median (interquartile range) UAER of 103 (39-230) $\mathrm{mg} / 24 \mathrm{~h}$ ). In addition to oral hypoglycaemic medications and insulin, most patients were treated with cardiovascular medication: antihypertensive drugs (99\%), RAAS-blocking treatment (98\%), statins (95\%) and aspirin (90\%).

ESM Tables 1 and 2 show the clinical characteristics of the study population according to quartiles of HGF and adiponectin, respectively (as potential confounders). For HGF, only NTproBNP differed significantly $(p=0.04)$ between quartiles (higher levels with increasing quartiles). For adiponectin, the levels of $\mathrm{HbA}_{1 \mathrm{c}}$, UAER and NT-proBNP were significantly higher with increasing quartiles $(p \leq 0.04)$.

\section{Incidence of the CVD endpoint and mortality}

The median follow-up was 6.1 years (IQR 5.9-6.6 years). During this period, 40 patients experienced at least one CVD endpoint and 26 patients died.

Eleven CVD events were fatal and 29 were non-fatal events leading to hospital admission, including two fatal and three non-fatal cases of acute myocardial infarction, three nonfatal strokes, one fatal and 19 non-fatal cases of ischaemic cardiovascular disease, six sudden and otherwise unexplained deaths, and two fatal and four non-fatal cases of heart failure. Of the 26 deaths, 11 were classified as CVD-related, nine as cancer-related, and six as related to other causes.

\section{Decline in the eGFR}

Patients with at least one annual eGFR measurement after baseline were included ( $n=177)$. The median (IQR) followup was 4.9 years (3.8-5.4 years). The eGFR declined from a mean $\pm \mathrm{SD}$ of $89.8 \pm 18.3 \mathrm{ml} \mathrm{min}^{-1} 1.73 \mathrm{~m}^{-2}$ at baseline to $79.1 \pm 21.4 \mathrm{ml} \mathrm{min}^{-1} 1.73 \mathrm{~m}^{-2}$ during follow-up $(p<0.001)$. A total of 42 patients (24\%) reached the pre-defined CKD progression endpoint. In 23 of these patients $(55 \%)$, the eGFR had declined by $>30 \%$ in at least two measurements during follow-up. However, for patients with a decline in eGFR in only one measurement (17 out of $19 ; \sim 89 \%$ ), this was seen for the first time at the final follow-up visit. Hence, it was not possible to confirm the eGFR decline of $>30 \%$ in these patients in a second test. However, based on the natural history of kidney disease in diabetes (which includes an expected annual decline in the eGFR), we do not think our findings reflect a transient fluctuation in the eGFR.

No patients reached end-stage renal disease or dialysis during follow-up.

\section{Urinary biomarkers}

Table 2 shows the associations for the urinary biomarkers with the composite CVD endpoint, all-cause mortality and CKD progression in all three models. 
Table 1 Clinical characteristics of the study population at baseline according to event status

\begin{tabular}{|c|c|c|c|c|c|c|}
\hline \multirow[t]{2}{*}{ Characteristic } & \multicolumn{2}{|l|}{ CVD event } & \multicolumn{2}{|l|}{ All-cause mortality } & \multicolumn{2}{|l|}{$\mathrm{CKD}_{\text {progression }}{ }^{\mathrm{a}}$} \\
\hline & No $(n=160)$ & Yes $(n=40)$ & No $(n=174)$ & Yes $(n=26)$ & No $(n=135)$ & Yes $(n=42)$ \\
\hline Male, $n(\%)$ & $115(72)$ & $36(90)$ & $129(74)$ & $22(85)$ & $125(93)$ & $32(76)$ \\
\hline Age, years & $58(9)$ & $63(6)$ & $58(9)$ & $62(8)$ & $58(8)$ & $60(7)$ \\
\hline Known duration of diabetes, years & $12(7)$ & $16(8)$ & $12(7)$ & $15(8)$ & $13(8)$ & $13(6)$ \\
\hline BMI, $\mathrm{kg} / \mathrm{m}^{2}$ & $32.7(5.8)$ & $31.9(5.8)$ & $32.6(5.7)$ & $32.4(6.4)$ & $32.6(5.7)$ & $32.5(6.0)$ \\
\hline $\mathrm{HbA}_{1 \mathrm{c}}, \%$ & $7.8(1.3)$ & $8.0(1.4)$ & $7.9(1.4)$ & $7.5(1.2)$ & $7.8(1.3)$ & $8.0(1.6)$ \\
\hline $\mathrm{HbA}_{1 \mathrm{c}}, \mathrm{mmol} / \mathrm{mol}$ & $62(14)$ & $64(15)$ & $63(15)$ & $59(13)$ & $63(16)$ & $63(13)$ \\
\hline UAER, mg/24 h & $100(38-209)$ & $119(44-509)$ & $96(38-200)$ & $196(52-510)$ & $96(36-192)$ & $143(51-692)$ \\
\hline Plasma creatinine, $\mu \mathrm{mol} / 1$ & $75(18)$ & $83(18)$ & $76(18)$ & $78(20)$ & $75(18)$ & $82(15)$ \\
\hline eGFR, $\mathrm{ml} \mathrm{min}{ }^{-1} 1.73 \mathrm{~m}^{-2}$ & $91.5(18.3)$ & $82.8(16.4)$ & $90.2(18.1)$ & $86.8(19.2)$ & $90.9(17.5)$ & $85.8(18.1)$ \\
\hline Systolic BP, mmHg & $130(16)$ & $130(15)$ & $130(16)$ & $130(18)$ & $129(16)$ & $132(14)$ \\
\hline LDL-cholesterol, mmol/1 & $1.8(0.8)$ & $2.0(0.8)$ & $1.8(0.8)$ & $1.9(0.8)$ & $1.9(0.8)$ & $1.8(0.7)$ \\
\hline Current smoker, $n(\%)$ & $45(28)$ & $14(35)$ & $45(26)$ & $14(54)$ & $42(31)$ & $9(21)$ \\
\hline NT-proBNP, ng/1 & $42.4(16.9-83.4)$ & $83.7(44.6-212.0)$ & $43.2(16.9-85.0)$ & $89.7(50.4-246.6)$ & $34.7(15.3-83.1)$ & $68.3(31.8-221.3)$ \\
\hline CAC score, Agatston units & $110(2-418)$ & $833(281-1,977)$ & $125(3-541)$ & $569(243-1,766)$ & $178(3-614)$ & $148(12-424)$ \\
\hline \multicolumn{7}{|l|}{ Treatment with } \\
\hline Oral antidiabetic, $n(\%)$ & $138(86)$ & $33(83)$ & $148(85)$ & $22(85)$ & $114(84)$ & $36(86)$ \\
\hline Insulin, $n(\%)$ & $94(59)$ & $30(75)$ & $106(61)$ & $17(65)$ & $82(61)$ & $24(57)$ \\
\hline Antihypertensive drugs, $n(\%)$ & $158(99)$ & $40(100)$ & $172(99)$ & $26(100)$ & $133(99)$ & $42(100)$ \\
\hline Statin, $n(\%)$ & $151(94)$ & $38(95)$ & $163(94)$ & $26(100)$ & $127(94)$ & $39(93)$ \\
\hline Aspirin, $n(\%)$ & $147(92)$ & $37(93)$ & $160(92)$ & $23(88)$ & $122(90)$ & $41(98)$ \\
\hline HGF, pg ml ${ }^{-1} \mathrm{~g}^{-1}$ creatinine & $13.4(8.6-17.1)$ & $16.5(12.6-20.1)$ & $13.3(8.9-17.0)$ & $18.0(13.2-22.1)$ & $13.0(8.9-17.0)$ & $15.9(11.5-19.4)$ \\
\hline Adiponectin, $\mathrm{pg} \mathrm{ml}^{-1} \mathrm{~g}^{-1}$ creatinine & $2.2(0.6-8.8)$ & $5.7(1.4-15.0)$ & $2.3(0.6-10.3)$ & $4.8(1.3-9.9)$ & $2.0(0.6-7.0)$ & $8.9(2.3-29.7)$ \\
\hline
\end{tabular}

Data are expressed as means (SD), median (interquartile range) or number of patients (\%)

${ }^{a}$ A total of 177 patients were available for the analyses of eGFR decline

Hepatocyte growth factor In Model 1, higher HGF was associated with both the composite CVD endpoint (HR 1.9 $[95 \%$ CI $1.3,2.8]$ ) and all-cause mortality (HR 2.3 [95\% CI 1.3, 3.9]; Table 2). In Model 2, higher HGF was associated with the composite CVD endpoint (HR 2.0 [95\% CI 1.2, 3.2]) and all-cause mortality (HR 2.5 [95\% CI 1.3, 4.8]). In Model 3 (Model 2 plus NT-proBNP and CAC), higher HGF remained associated with both the composite CVD endpoint (HR 1.5 $[95 \%$ CI 1.1, 2.3]) and all-cause mortality (HR 1.9 [95\% CI $1.0,3.5])$.

Table 2 Urinary biomarkers related to risk of fatal and non-fatal CVD event, all-cause mortality and CKD progression

\begin{tabular}{|c|c|c|c|c|c|c|c|}
\hline \multirow[t]{2}{*}{ Urinary biomarker } & \multirow[t]{2}{*}{ Model } & \multicolumn{2}{|c|}{ CVD event $(n=40)$} & \multicolumn{2}{|c|}{ All-cause mortality $(n=26)$} & \multicolumn{2}{|c|}{ CKD progression $(n=42)$} \\
\hline & & $\operatorname{HR}(95 \% \mathrm{CI})$ & $p$ value & HR $(95 \% \mathrm{CI})$ & $p$ value & HR $(95 \%$ CI $)$ & $p$ value \\
\hline \multirow[t]{3}{*}{ HGF, log scale $(1 \mathrm{SD}=0.22)$} & 1 & $1.9(1.3,2.8)$ & 0.001 & $2.3(1.3,3.9)$ & 0.003 & $1.4(0.9,2.1)$ & 0.11 \\
\hline & 2 & $2.0(1.2,3.2)$ & 0.004 & $2.5(1.3,4.8)$ & 0.005 & $1.1(0.7,1.7)$ & 0.81 \\
\hline & 3 & $1.5(1.1,2.3)$ & 0.026 & $1.9(1.0,3.5)$ & 0.034 & - & \\
\hline \multirow[t]{3}{*}{ Adiponectin, log scale $(1 \mathrm{SD}=0.74)$} & 1 & $1.4(1.0,1.9)$ & 0.040 & $1.1(0.7,1.6)$ & 0.53 & $1.6(1.2,2.2)$ & 0.008 \\
\hline & 2 & $1.4(1.1,2.3)$ & 0.013 & $1.2(0.7,2.0)$ & 0.41 & $1.5(1.1,2.2)$ & 0.007 \\
\hline & 3 & $1.4(0.9,2.2)$ & 0.18 & $1.1(0.6,2.2)$ & 0.59 & - & \\
\hline
\end{tabular}

Values represent an SD increment of log-transformed values of the biomarkers

Model 1 is unadjusted

Model 2 is adjusted for sex, age, LDL-cholesterol, smoking, $\mathrm{HbA}_{1 \mathrm{c}}$, plasma creatinine, systolic BP and UAER

Model 3 (for cardiovascular events and all-cause mortality) is Model 2 plus adjustment for CAC score and NT-proBNP level 
When dividing patients into two groups according to level of HGF (above or below the median [14.1 $\mathrm{pg} \mathrm{ml}^{-1} \mathrm{~g}^{-1}$ creatinine]), the unadjusted risk of the composite CVD endpoint (HR 2.5 [95\% CI 1.2, 4.8]) and all-cause mortality (HR 2.6 $[95 \%$ CI 1.1, 6.2]) was higher in patients with levels above the median (Fig. 2).

As shown in ESM Fig. 2a,b, in relation to the composite CVD endpoint, the AUC changed from 0.738 (95\% CI 0.660 , $0.816)$ to $0.775(95 \%$ CI $0.698,0.853)$ after HGF was added to the model including traditional $\mathrm{CVD} /$ renal risk factors (change in AUC 0.0374 [95\% CI -0.003, 0.078]; $p=0.072$ ). In relation to all-cause mortality, the AUC changed from 0.746 (95\% CI

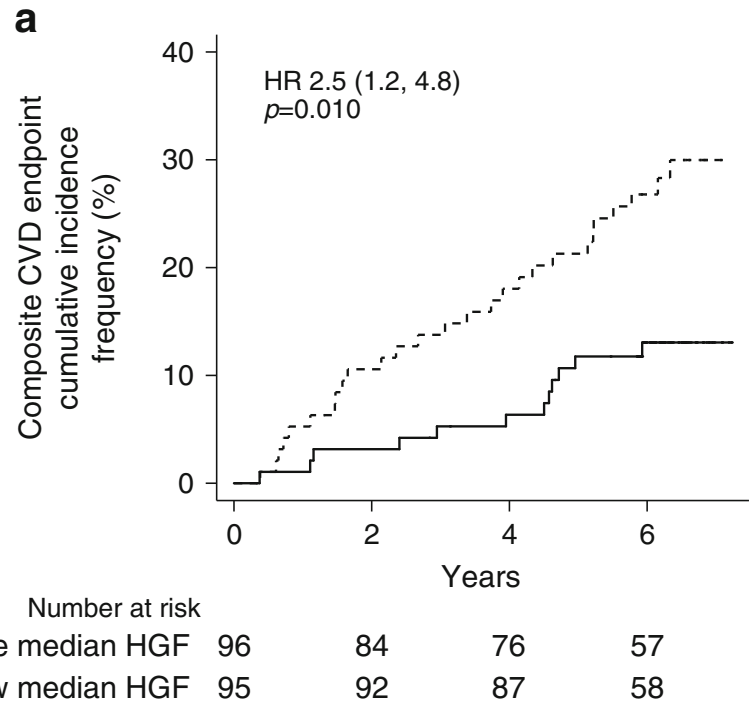

$\begin{array}{lllll}\text { Above median HGF } & 96 & 84 & 76 & 57 \\ \text { Below median HGF } & 95 & 92 & 87 & 58\end{array}$

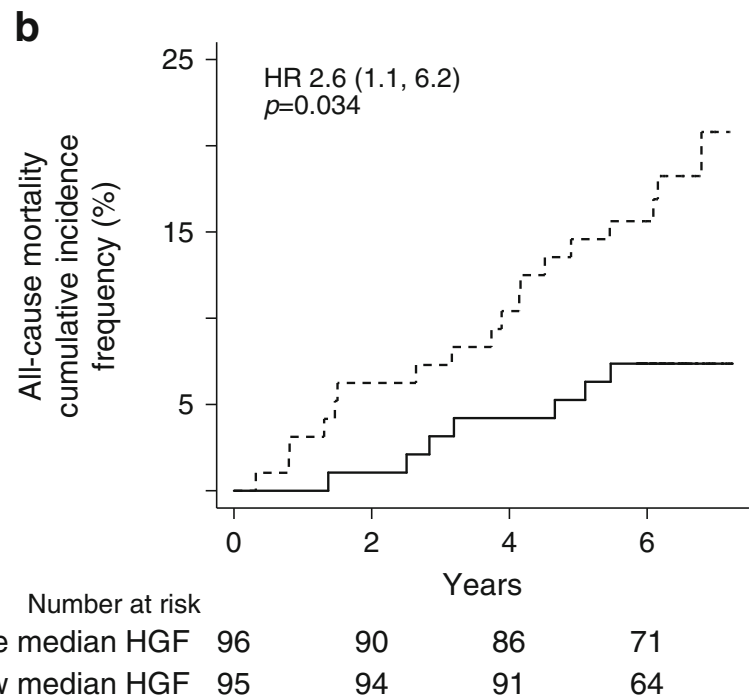

$\begin{array}{lllll}\text { Above median HGF } & 96 & 90 & 86 & 71 \\ \text { Below median HGF } & 95 & 94 & 91 & 64\end{array}$

Fig. 2 Proportion of type 2 diabetic patients experiencing incident CVD and all-cause mortality related to higher and lower levels of urinary HGF. Kaplan-Meier failure function estimates for (a) the composite cardiovascular endpoint and (b) all-cause mortality in two categories of the levels of urinary HGF. HR is shown with $95 \%$ CI for both endpoints. Numbers refer to participants in each category at risk at the beginning of each 2 year interval. Dashed line, above median HGF; solid line, below median HGF
$0.645,0.846)$ to $0.774(95 \%$ CI $0.663,0.884)$ after adding HGF to the model including traditional $\mathrm{CVD} /$ renal risk factors (change in AUC 0.0280 [95\% CI -0.046, 0.102]; $p=0.46$ ).

The rIDI for HGF was $29.5 \%$ (95\% CI 13.7\%, 45.3\%; $p=0.005)$ in relation to the CVD endpoint and $87.1 \%$ $(50.0 \%, 123.8 \% ; p<0.001)$ in relation to all-cause mortality.

Levels of HGF were not related to the CKD progression endpoint in Model 1 (HR 1.4 [95\% CI 0.9, 2.1]) or Model 2 (HR 1.1 [95\% CI 0.7, 1.7]).

Adiponectin In Model 1, higher adiponectin was associated with the composite CVD endpoint (HR 1.4 [95\% CI 1.0, 1.9]), but was not associated with all-cause mortality (HR 1.1 [95\% CI $0.7,1.6])$. Higher levels of adiponectin were also associated with the composite CVD endpoint (HR 1.4 [95\% CI 1.1, 2.3]) in Model 2. However, in Model 3, when NT-proBNP and $\mathrm{CAC}$ were included in the adjustment, adiponectin lost significance and was not associated with the composite CVD endpoint (HR 1.4 [95\% CI 0.9, 2.2]).

As shown in ESM Fig. 2c,d, in relation to the composite CVD endpoint, the AUC changed from 0.738 (95\% CI 0.660 , $0.816)$ to $0.763(95 \% \mathrm{CI} 0.687,0.839)$ after adding adiponectin to the model including traditional $\mathrm{CVD} /$ renal risk factors (change in AUC 0.025 [95\% CI -0.014, 0.061]; $p=0.22$ ).

In relation to all-cause mortality, the AUC changed from 0.746 (95\% CI $0.645,0.846)$ to 0.761 (95\% CI $0.662,0.859)$ after adding adiponectin to the model including traditional CVD/renal risk factors (change in AUC 0.015 [95\% CI $-0.022,0.042] ; p=0.65)$.

The rIDI for adiponectin was $14.4 \%(95 \% \mathrm{CI}-1.6 \%, 29.6 \%$; $p=0.054)$ in relation to the CVD endpoint, and $8.9 \%(95 \% \mathrm{CI}$ $-21.1 \%, 40.2 \% ; p=0.26)$ in relation to all-cause mortality.

In Model 1, higher levels of adiponectin were associated with decline in the eGFR of $>30 \%$ (HR 1.6 [95\% CI 1.2, 2.2]). In Model 2, higher levels of adiponectin remained associated with a decline in the eGFR of $>30 \%$ (HR 1.5 [95\% CI 1.1, 2.2]).

\section{Additional analyses}

In the Cox models with adjustment based on backward selection, higher levels of HGF were associated with the composite CVD endpoint (HR 1.9 [95\% CI 1.2, 2.9]; $p=0.003$ ) and allcause mortality (HR 2.4 [95\% CI 1.3, 4.2]; $p=0.003$ ); higher levels of adiponectin were associated with the composite CVD endpoint (HR 1.4 [95\% CI 1.0, 2.0]; $p=0.037$ ) and the CKD progression endpoint (HR 1.6 [95\% CI 1.1, 2.2]; $p=0.010$ ).

As novel biomarkers may not be as accessible to clinicians, we also sought to determine whether similar findings were obtained for risk indicators that are already available (i.e. albuminuria). Therefore, we evaluated the rate of change in degree of albuminuria prior to baseline in relation to risk of CVD, all-cause mortality and decline in the eGFR. A greater increase in albuminuria was related to all-cause mortality in 
unadjusted and adjusted analysis $(p \leq 0.008)$, but not to CVD or a decline in the eGFR ( $\mathrm{p} \geq 0.33)$.

\section{Discussion}

In this prospective study of patients with type 2 diabetes and microalbuminuria with no clinical features of $\mathrm{CAD}$ and who were receiving multifactorial treatment, we have demonstrated HGF to be an independent determinant of incident CVD and allcause mortality after 6 years of follow-up. Even when accounting for levels of NT-proBNP and CAC, higher HGF was associated with both endpoints. Higher adiponectin was a determinant of the CVD endpoint and was associated with CKD progression (defined as a reduction in the eGFR of at least 30\%), even when baseline levels of creatinine and albuminuria were accounted for. An analysis of the added discriminatory ability of the biomarkers showed no significant increase in the AUC for incident CVD or all-cause mortality when each biomarker was added to models that included traditional CVD risk factors. However, the IDI statistics (considered a more powerful way to demonstrate improvement [18]) showed that the addition of HGF significantly improved the predictive capability for both CVD and all-cause mortality. For adiponectin the increase in rIDI was borderline significant for the CVD endpoint.

Knowledge of the aetiology of type 2 diabetes and its associated vascular complications has increased considerably over recent years. Biomarkers reflecting the well-accepted pathophysiologic pathways are important markers of diabetic complications, and may provide targets for therapy. The beneficial effect of multifactorial treatment was demonstrated in the Steno 2 Study [13]. Despite this, patients with type 2 diabetes, especially those with albuminuria, are still at a high risk of complications [19].

In the current cohort, we have recently demonstrated that a simple screening algorithm that combines the NT-proBNP level and CAC score added substantially to the information provided by traditional CVD risk factors [17]. Even after adjustment for these powerful traditional risk markers, higher levels of HGF remained associated with the composite CVD endpoint and all-cause mortality.

HGF is a pleiotropic factor that has a wide variety of roles in diverse biological processes, such as organ development, tissue homeostasis and injury repair [20]. Studies also indicate that treatment with HGF has therapeutic effects for patients with chronic renal disease [21].

HGF is upregulated in the kidneys after acute kidney injury, and administration of exogenous HGF promotes tubular repair and recovery in rats [22]. Endogenous activation of HGF tends to preserve kidney structure and function in rats with chronic renal disease [23]. Few cross-sectional studies have investigated the relationship between serum levels of HGF and atherosclerotic involvement. A single population- based study showed a cross-sectional relationship between serum levels of HGF and the presence of retinal arteriosclerotic lesions [24]. In patients with end-stage renal disease, studies have demonstrated that serum HGF levels correlate with carotid intima-media thickness and concentric left ventricular geometry [8]. However, to the best of our knowledge, we are the first to show an association between levels of urinary HGF and both incident CVD and all-cause mortality in type 2 diabetes. Importantly, the addition of HGF to traditional cardiovascular risk factors added predictive value for both endpoints.

Adiponectin has a wide range of well-known protective effects against insulin resistance, vascular dysfunction, atherosclerosis and inflammation [25], but has also been shown to be important for maintaining healthy podocytes [26]. A crosssectional study by von Eynatten et al demonstrated that higher urinary adiponectin levels correlated significantly with increased intima-media thickness in type 2 diabetes [7]. In our cohort, we extended these findings to show that levels of urinary adiponectin were associated with incident CVD and CKD progression. In relation to serum adiponectin, high levels have been linked to cardiovascular mortality in men with type 2 diabetes [27]. Moreover, we and others have previously demonstrated that high serum adiponectin levels predict all-cause mortality, cardiovascular mortality and progression to endstage renal disease in patients with type 1 diabetes [28, 29]. Of note, our pre-defined CKD progression endpoint was a decline in the eGFR of $>30 \%$ at any time point. We considered that this is appropriate because our cohort included microalbuminuric patients, and decreases in the eGFR associated with less than a doubling of serum creatinine have recently been shown to be strongly and consistently associated with the risk of end-stage renal disease and mortality in a large metaanalysis [16]. Therefore, a smaller decline in the eGFR (such as a $30 \%$ reduction) has been suggested as an alternative endpoint for CKD progression [16]. Lastly, the European Medicines Agency has proposed a clinically relevant delay in milder renal function loss (e.g. loss of $30 \%$ in the eGFR) as a potential endpoint in clinical trials investigating new products [30].

\section{Clinical implications}

Measuring urinary HGF and adiponectin is relatively uncomplicated and inexpensive. Our findings illustrate that these biomarkers could improve risk assessment for type 2 diabetic patients. However, our findings need to be validated. Identifying patients at elevated risk of CVD and all-cause mortality is crucial because these patients may benefit from even tighter goal setting for traditional risk factors, such as BP or LDL-cholesterol. Moreover, intervention studies might show whether treating HGF and adiponectin is of clinical benefit in terms of risk reduction for individual patients. 


\section{Strengths and limitations}

The study strengths were that no patients were lost to follow-up and our analysis allowed extensive adjustment for important risk factors. However, there were limitations. Although the sample size and number of events were limited, the CVD event rate was as expected based on our sample size calculation. In addition, although the statistical model might be vulnerable to instability due to overfitting, additional analyses to confirm the robustness of our findings indicated stability. We lacked information on how long the patients had received multifactorial treatment before the baseline blood sampling and to what extent they effectively received such treatment during follow-up. Nevertheless, attending physicians generally follow our local guidelines on intensive multifactorial treatment for individuals with type 2 diabetes and microalbuminuria, based on the Steno-2 study findings [13]. Finally, serum levels of HGF and adiponectin were not measured in this study.

\section{Conclusion}

In patients with type 2 diabetes and microalbuminuria but without clinical features of $\mathrm{CAD}$ who were receiving multifactorial treatment, levels of urinary HGF were associated with incident CVD and all-cause mortality, even after adjustment for albuminuria, NT-proBNP level and $\mathrm{CAC}$ score. Higher adiponectin was a determinant of the CVD endpoint and was associated with CKD progression, even when accounting for baseline creatinine and albuminuria.

Acknowledgements We thank A. Horsch, Business Development, Markers \& Disease Area Strategies, Roche Diagnostics, Rotkreuz, Switzerland, for measuring the two urinary biomarkers investigated. We thank E. G. Noctor, Steno Diabetes Center, Gentofte, Denmark, for linguistic help and proofreading the manuscript. We also thank all participants and acknowledge the work of L. Jelstrup (the study nurse) and A.G. Lundgaard, B. R. Jensen, T. R. Juhl and J. A. Hermann (laboratory technicians), Steno Diabetes Center, Gentofte, Denmark.

Funding Internal funding was provided by Steno Diabetes Center, Gentofte, Denmark.

Duality of interest statement The authors declare that there is no duality of interest associated with this manuscript.

Contribution statement BJvS, HR, H-HP, PKJ and PR conceived and designed the research; BJvS, HR, PR and TWH analysed and interpreted the data; BJvS and JO performed the statistical analysis; BJvS wrote the manuscript; HR, H-HP, JO, PKJ and TWH critically revised the manuscript for key intellectual content; and PR obtained funding and supervised the study. All authors approved the final version of the manuscript. $\mathrm{BJvS}$ is responsible for the integrity of the work as a whole.

\section{References}

1. de Zeeuw D, Remuzzi G, Parving H-H et al (2004) Proteinuria, a target for renoprotection in patients with type 2 diabetic nephropathy: lessons from RENAAL. Kidney Int 65:2309-2320

2. Lorenzo V, Saracho R, Zamora J, Rufino M, Torres A (2010) Similar renal decline in diabetic and non-diabetic patients with comparable levels of albuminuria. Nephrol Dial Transplant 25:835-841

3. Gerstein HC, Mann JF, Yi Q et al (2001) Albuminuria and risk of cardiovascular events, death, and heart failure in diabetic and nondiabetic individuals. JAMA 286:421-426

4. Valmadrid CT, Klein R, Moss SE, Klein BE (2000) The risk of cardiovascular disease mortality associated with microalbuminuria and gross proteinuria in persons with older-onset diabetes mellitus. Arch Intern Med 160:1093-1100

5. Yuyun MF, Dinneen SF, Edwards OM, Wood E, Wareham NJ (2003) Absolute level and rate of change of albuminuria over 1 year independently predict mortality and cardiovascular events in patients with diabetic nephropathy. Diabet Med 20:277-282

6. Liu Y, Yang J (2006) Hepatocyte growth factor: new arsenal in the fights against renal fibrosis? Kidney Int 70:238-240

7. von Eynatten M, Liu D, Hock C et al (2009) Urinary adiponectin excretion: a novel marker for vascular damage in type 2 diabetes. Diabetes 58:2093-2099

8. Malatino LS, Cataliotti A, Benedetto FA et al (2003) Hepatocyte growth factor and left ventricular geometry in end-stage renal disease. Hypertension 41:88-92

9. Jorsal A, Petersen EH, Tarnow L et al (2013) Urinary adiponectin excretion rises with increasing albuminuria in type 1 diabetes. J Diabetes Complicat 27:604-608

10. Fufaa GD, Weil EJ, Nelson RG et al (2015) Association of urinary KIM-1, L-FABP, NAG and NGAL with incident end-stage renal disease and mortality in American Indians with type 2 diabetes mellitus. Diabetologia 58:188-198

11. Panduru NM, Saraheimo M, Forsblom C et al (2015) Urinary adiponectin is an independent predictor of progression to endstage renal disease in patients with type 1 diabetes and diabetic nephropathy. Diabetes Care 38:883-890

12. Reinhard H, Hansen PR, Persson F et al (2011) Elevated NT-proBNP and coronary calcium score in relation to coronary artery disease in asymptomatic type 2 diabetic patients with elevated urinary albumin excretion rate. Nephrol Dial Transplant 26:3242-3249

13. Gaede P, Lund-Andersen H, Parving HH, Pedersen O (2008) Effect of a multifactorial intervention on mortality in type 2 diabetes. N Engl J Med 358:580-591

14. Agatston AS, Janowitz WR, Hildner FJ, Zusmer NR, Viamonte M Jr, Detrano R (1990) Quantification of coronary artery calcium using ultrafast computed tomography. J Am Coll Cardiol 15:827-832

15. Levey AS, Stevens LA, Schmid CH et al (2009) A new equation to estimate glomerular filtration rate. Ann Intern Med 150:604-612

16. Coresh J, Turin TC, Matsushita K et al (2014) Decline in estimated glomerular filtration rate and subsequent risk of end-stage renal disease and mortality. JAMA 311:2518-2531

17. von Scholten BJ, Reinhard H, Hansen TW et al (2015) Additive prognostic value of plasma $\mathrm{N}$-terminal pro-brain natriuretic peptide and coronary artery calcification for cardiovascular events and mortality in asymptomatic patients with type 2 diabetes. Cardiovasc Diabetol 14:59

18. Pencina MJ, D'Agostino RB, Vasan RS (2010) Statistical methods for assessment of added usefulness of new biomarkers. Clin Chem Lab Med 48:1703-1711

19. de Zeeuw D, Parving HH, Henning RH (2006) Microalbuminuria as an early marker for cardiovascular disease. J Am Soc Nephrol 17: 2100-2105 
20. Zhou D, Tan RJ, Lin L, Zhou L, Liu Y (2013) Activation of hepatocyte growth factor receptor, c-met, in renal tubules is required for renoprotection after acute kidney injury. Kidney Int 84:509-520

21. Matsumoto K, Nakamura T (2002) Renotropic role and therapeutic potential of HGF in the kidney. Nephrol Dial Transplant 17(Suppl 9):59-61

22. Liu Y, Tolbert EM, Lin L et al (1999) Up-regulation of hepatocyte growth factor receptor: an amplification and targeting mechanism for hepatocyte growth factor action in acute renal failure. Kidney Int 55: 442-453

23. Liu Y, Rajur K, Tolbert E, Dworkin LD (2000) Endogenous hepatocyte growth factor ameliorates chronic renal injury by activating matrix degradation pathways. Kidney Int 58:2028-2043

24. Nishimura M, Ushiyama M, Nanbu A, Ohtsuka K, Takahashi H, Yoshimura M (1997) Serum hepatocyte growth factor as a possible indicator of arteriosclerosis. J Hypertens 15:1137-1142

25. Li FY, Cheng KK, Lam KS, Vanhoutte PM, Xu A (2011) Cross-talk between adipose tissue and vasculature: role of adiponectin. Acta Physiol 203:167-180
26. Sharma K, Ramachandrarao S, Qiu G et al (2008) Adiponectin regulates albuminuria and podocyte function in mice. J Clin Invest 118:1645-1656

27. Menzaghi C, Xu M, Salvemini L et al (2014) Circulating adiponectin and cardiovascular mortality in patients with type 2 diabetes mellitus: evidence of sexual dimorphism. Cardiovasc Diabetol 13:130

28. Jorsal A, Tarnow L, Frystyk J et al (2008) Serum adiponectin predicts all-cause mortality and end stage renal disease in patients with type I diabetes and diabetic nephropathy. Kidney Int 74:649-654

29. Forsblom C, Thomas MC, Moran J et al (2011) Serum adiponectin concentration is a positive predictor of all-cause and cardiovascular mortality in type 1 diabetes. J Intern Med 270:346-355

30. Committee for Medicinal Products for Human Use (2014) Guideline on the clinical investigation of medicinal products to prevent development/slow progression of chronic renal insufficiency. European Medicines Agency, www.ema.europa.eu/docs/en $\mathrm{GB} /$ document library/Scientific guideline/2014/06/ WC500169469.pdf, accessed 1 Aug $201 \overline{5}$ 